\title{
Filosofía como forma de vida y práctica filosófica en Colombia: una aproximación al estado del arte*
}

\author{
Philosophy as a form of life and philosophical practice in \\ Colombia: an approach to the state of art
}

Filosofia como forma de vida e pratica filosófica na Colômbia: uma aproximação ao estado da arte

\author{
Johana Páez Vigoya** \\ Andrés Felipe Urrego Salas ${ }^{* *}$
}

\section{Resumen}

Este documento pretende explorar las categorías de filosofía como forma de vida -como la plantea Pierre Hadot-y de práctica filosófica a partir del rastreo de algunos de los trabajos más relevantes de autores colombianos que se han destacado en la investigación al respecto.

* El texto presenta un estudio de caso que los autores adelantaron como investigación para la obtención de su grado de licenciados en Filosofia. DOI:http://dx.doi.org/10.15332/s0120-8462.2017.0116.07

* Licenciada en Filosofía de la Universidad Pedagógica Nacional de Colombia. Miembro del grupo de investigación Filosofía y Enseñanza de la Filosofia. Correo electrónico: jpaezvigoya@gmail.com

*** Estudiante de la Licenciatura en Filosofía de la Universidad Pedagógica Nacional de Colombia. Miembro del grupo de investigación Filosofía y Enseñanza de la Filosofía. Correo electrónico: afelipeurrego@ gmail.com 
Principalmente se encuentran tres categorías de investigación en las que se distinguen reflexiones acerca de la teoría hadotiana, sobre la tradición filosófica a la luz de tal teoría y trabajos sobre problemas educativos y prácticas pedagógicas realizadas desde dicha perspectiva. Se propone la relación entre la filosofía como forma de vida y la práctica filosófica, especialmente, desde el campo educativo. El análisis realizado permite ver posibles líneas de investigación y los ejes temáticos con los que las mismas se plantearían.

Palabras clave: forma de vida, práctica filosófica, Colombia, educación, filosofía.

\section{Abstract}

This document aims to explore the categories of philosophy as a form of life -as Pierre Hadot proposes- and of philosophical practice from the tracing of some of the most relevant works of Colombian authors who have excelled in the research on the subject. Mainly three research categories are found which distinguish reflections on the Hadotian theory on the philosophical tradition in the light of such theory and works on educational problems and pedagogical practices made from that perspective. The relationship between philosophy as a form of life and philosophical practice is proposed, especially from the educational field. The analysis made allows to see possible lines of research and the thematic axes with which they would be presented.

Keywords: form of life, philosophical practice, Colombia, education, philosophy. 


\section{Resumo}

Este documento pretende explorar as categorias de filosofia como forma de vida-como o expõe Pierre Hadot- e de pratica filosófica a partir do rastreio de alguns dos trabalhos mais relevantes de autores colombianos que se destacam nas pesquisas sobre o tema. Principalmente existem três categorias de pesquisa nas quais distinguem reflexões sobre a teoria hadotiana, sobre a tradição filosófica à luz de essa teoria e de trabalhos sobre problemas educativos e praticas pedagógicas realizadas desde essa perspectiva. Propõe-se a relação entre a filosofia como forma de vida e a pratica filosófica, especialmente desde o campo educativo. A análise realizada permite ver possíveis linhas de pesquisa e os eixos temáticos com os que as mesmas se apresentaram.

Palavras-chave: forma de vida, prática filosófica, Colômbia, educação, filosofia. 


\section{Introducción}

Al plantear una filosofía como forma de vida se establece un compromiso con la concepción que se tiene de la filosofía, pues en este sentido se entiende como un ejercicio formativo para la construcción del ser humano y no como una disciplina meramente teórica. Durante el proceso de la investigación que emprendimos pudimos reconocer que dicho tema se ha abordado desde varios pensadores y desde múltiples perspectivas. El análisis que presentamos en este documento está centrado en esta percepción de la filosofía a partir del estudio de algunas obras del filósofo francés Pierre Hadot (1998-2006), quien planteó su propuesta partiendo principalmente de sus estudios sobre la filosofía antigua. La perspectiva que plantea Hadot aboga por una filosofía que tenga eco en la vida del ser humano estableciendo una relación con la formación del mismo desde prácticas específicas.

Ahora bien, pudimos encontrar tres ámbitos en los que es posible identificar esta visión de la filosofía: primero, el trabajo de Pierre Hadot desde un estudio principalmente teórico; segundo, teniendo en cuenta una perspectiva relacionada con el ejercicio práctico lo situamos en el movimiento denominado práctica filosófica; tercero, en relación con el ámbito educativo. Se retomará la práctica filosófica a partir de la perspectiva de Gerd Achenbach, quien esboza una propuesta que versa sobre prácticas y actividades aplicadas dentro y fuera de la academia formal. La práctica filosófica es en gran medida influenciada por Hadot y su caracterización de la filosofía en la antigua Grecia.

El vínculo que pudimos establecer entre los tres enfoques mencionados se da en tanto que la práctica filosófica la entendemos como una ruptura entre la teoría y la praxis, lo cual da cuenta de la forma en que la filosofía implica una serie de prácticas que conducen a la transformación de la vida misma.

En Colombia existen trabajos y propuestas que caben dentro de las perspectivas enunciadas, por lo que consideramos necesario hacer un estudio sobre cómo se ha llevado esta labor en el país. Para tal propósito, analizamos las principales investigaciones: trabajos de grado, tesis, grupos de investigación, artículos, libros, eventos, revistas; las cuales se han elaborado en el contexto nacional a la luz de los dos primeros enfoques propuestos. Se revisaron publicaciones colombianas como la revista Práxis filosófica de la Universidad del Valle, la revista Ideas y valores de la Universidad Nacional de Colombia, la revista Universitas philosophica de la Pontificia Universidad Javeriana, 
la revista Cuestiones de filosofía, de la Universidad Pedagógica y Tecnológica de Colombia, la revista Franciscanum de la Universidad San Buenaventura, la revista Versiones y la revista Estudios de filosofía de la Universidad de Antioquia. Asimismo, se revisaron eventos y grupos de investigación en plataformas como GrupLAC y CvLAC, Academia.edu y SciELO.

A lo largo del presente trabajo es transversal la exploración sobre las principales publicaciones en las que es notorio el vínculo de la filosofía como forma de vida y la práctica filosófica con la educación. Esto para reconocer los alcances pedagógicos que ha tenido esta propuesta y que podrían darse a futuro, no solo por el carácter formativo que se le atribuye a la filosofía como forma de vida sino también por los intereses del proyecto de investigación.

\section{El concepto de filosofía como forma de vida}

Pierre Hadot (2006), en sus estudios acerca de la filosofía antigua grecorromana, muestra que esta tiene una característica particular, a saber, que implica una actitud moral y una forma específica de estar en el mundo. Para los antiguos, encaminarse en los rumbos de la filosofía supone adquirir una forma de vivir; la filosofía envuelve una actividad que lleva a transformar la existencia en su conjunto. Esta perspectiva de los antiguos corresponde a una serie de prácticas que Hadot llama ejercicios espirituales. La filosofía es un ejercitarse en el que se adquirieren prácticas que irrumpen en los modos habituales de vivir. Esto último incluye no solo un ámbito referido únicamente al pensamiento (razón por la que se usa el término espiritual) sino que comprende un campo más amplio del vivir humano:

La palabra "espiritual" permite comprender con mayor facilidad que unos ejercicios como estos son producto no solo del pensamiento, sino de una totalidad psíquica del individuo que, en especial, revela el auténtico alcance de tales prácticas: gracias a ellas el individuo accede al círculo del espíritu objetivo, lo que significa que vuelva a situarse en la perspectiva del todo (Hadot, 2006, p. 24).

Concebir la filosofía como una práctica constituye un arte de la existencia para aprender a vivir. Hadot expone cómo las diferentes escuelas filosóficas de la antigüedad se forman desde esta posición, cada una con sus propios principios como un método para alcanzar la independencia y la libertad interior, buscando curar la angustia que 
traería la falta de las mismas; la filosofía en este sentido implica también una labor terapéutica:

\begin{abstract}
Se trata de una forma de existencia caracterizada por tres aspectos fundamentales: la paz espiritual (ataraxia), la libertad interior (autarkeia) y, salvo en el caso de los escépticos, la consciencia cósmica, es decir, esa consciencia de pertenencia al todo humano y cósmico consistente en una suerte de dilatación, de transfiguración del yo gracias al cual se consigue la grandeza de alma (megalopsuchia) (Hadot, 2006, p. 254).
\end{abstract}

Entre tanto, el discurso filosófico se emplea en estas escuelas con propósitos de enseñanza. De modo que los principios de cada escuela se configuran en un discurso que no solo les suma un carácter persuasivo, sino que también posibilitan su memorización, y, sobre todo, su aplicación. Así pues, lo terapéutico se confiere también a la palabra. Los ejercicios terapéuticos, en este sentido, se dan en medio de diálogos con otros y consigo mismo, tomando como vehículo para tanto la escritura como la lectura. De esta manera, se busca un dominio de sí mismo: “Quien desee progresar tiene que esforzarse por dirigir ordenadamente sus pensamientos, alcanzando así una transformación completa de su representación del mundo, de su paisaje interior, pero al mismo tiempo de su comportamiento exterior" (Hadot, 2006, p. 29).

Asimismo, la meditación es acompañada de la lectura de textos poéticos y filosóficos, así como de la escucha de enseñanzas de maestros; los ejercicios tienen el propósito de crear hábitos. Hadot (1998), citando a Séneca, por ejemplo, muestra cómo la meditación sirve para anticiparse a problemas como la pobreza y el sufrimiento, para prepararse en caso de imprevistos de este tipo. Imprevistos que al no depender de nosotros no se pueden tomar como males. Se meditan cada día las actividades a realizar planteando principios que las guiarán, posteriormente se analizan de nuevo para revisar faltas y progresos (Hadot, 2006, p. 29). La distancia entre el discurso filosófico y la vida filosófica es que esta última implica una transformación de sí mismo, el discurso es apenas parte de ello.

\title{
Estudios sobre la propuesta de Hadot
}

En Colombia se han desarrollado estudios sobre la obra de Hadot y de la forma en que concibió la filosofía. El trabajo de este autor permitió el inicio de investigaciones 
sobre su propuesta y a partir de ella nuevas interpretaciones de las obras de pensadores clásicos. En Colombia podemos encontrar investigaciones en las que se ha emprendido la labor de estudio y comprensión de la obra de Hadot. A continuación, expondremos algunas de estas.

Germán Meléndez (2015) hace una aproximación a la noción de filosofía como forma de vida, a partir de la relación entre el discurso filosófico y la forma de vida defendidos por Hadot. Para lograrlo, caracteriza el discurso filosófico con el propósito de mostrar en qué sentido dicho pensamiento posee un carácter discursivo. Igualmente, identifica la actividad filosófica como una constante investigación, en tanto está supeditada a un qué y un cómo que justifica su labor. Posteriormente, Meléndez hace referencia a la noción de forma de vida, en la que Hadot remite a una manera en la que se actúa y se comprende el mundo, en el que hay una coherencia entre el discurso y la práctica. Aunque Hadot muestre en principio que es la forma de vida la que posibilita el discurso filosófico, y no al contrario, Meléndez evidencia que Hadot concibe que ambas nociones (discurso y forma de vida) se relacionan de manera recíproca y no solo de forma unidireccional; así el discurso procede de manera retroactiva sobre la forma de vida elegida, justificándola racionalmente. De igual manera, el discurso filosófico se puede asociar con un ejercicio, una práctica que posibilita la formación y la transformación de otros y de sí mismo.

Además de hacer una reconstrucción de la propuesta de Hadot, Damián Pachón Soto (2014) muestra que Wittgenstein fue de gran influencia para el autor francés, pues le permitió ver que el ejercicio filosófico se da entre "juegos del lenguaje" o "formas de vida", lo que implica que el uso del lenguaje tiene que ver con los contextos. De lo anterior se infiere que los textos filosóficos deben ponerse en perspectiva con los contextos en que se produjeron. Pachón Soto también denota que esta forma de hacer filosofía no murió en la antigüedad y rescata la obra de autores como Francis Bacon y Montaigne. El autor también destaca el trabajo de autores como Michel Onfray, Peter Sloterdijk y Michel Foucault, quienes han hecho estudios por vías similares a las de Hadot.

Jorge Aurelio Díaz (2001), por otro lado, también hace mención a la transición de la filosofía como sierva de la teología en el medioevo, en donde su tarea se fue relegando más al ejercicio teórico que al práctico; es decir, la orientación de la vida justificada a la religión y no al ejercicio filosófico. Asimismo, Díaz expone que la filosofía en la modernidad, aunque se aleja del mero servicio a la teología, se presta para servir a 
todos los campos del saber, aunque también reconoce que de nuevo empieza a retornar hacia sí misma determinando sus objetivos propios. Para ejemplificar esto último, Díaz hace una distinción entre los propósitos filosóficos de Descartes y Spinoza, en donde el primero busca un conocimiento firme, y el segundo, tras sentar unas bases epistemológicas, llega a la ética para justificar la felicidad. Finalmente se muestra cómo en la actualidad hay corrientes (con autores como Heidegger y Foucault) que abogan por una autocreación en el ejercicio filosófico en contraposición a quienes abogan por una filosofía al servicio de otras causas y saberes, que Díaz describe como una labor subsidiaria.

En Colombia también existe un trabajo de traducción emprendido por Fernando Alba del texto de Michel Chase Recordando a Pierre Hadot (2012). En este escrito se rinde un homenaje al filósofo francés tras su muerte, haciendo un reconocimiento de su labor a nivel personal, docente, investigativa y filosófica. También podemos localizar la labor del grupo Peiras (grupo de investigación de la Universidad Nacional de Colombia y la Universidad de los Andes, cuyo enfoque de investigación es la filosofía antigua y medieval), el cual comenzó un proyecto llamado Filosofía como forma de vida.

En gran parte de las publicaciones de Peiras predomina un estudio de casos particulares de filósofos y textos filosóficos en donde se rastrea la noción de filosofía como forma de vida. El grupo también impartió en la Universidad Nacional de Colombia el curso de extensión titulado ¿Convertir la vida en arte? Una breve historia de la filosofía como forma de vida en el segundo semestre del año 2013, a cargo del docente Germán Meléndez. El curso tuvo como objetivo hacer una introducción al tema en cuestión haciendo un recorrido desde la filosofía antigua hasta la filosofía contemporánea.

\section{El estudio de la tradición filosófica en relación con la filosofía como forma de vida}

En el país también existen trabajos que se estructuran desde una perspectiva menos general y más enfocada en el estudio de casos particulares de obras de la filosofía que despliegan una visión práctica y vital del ser humano. En su mayoría resultan de investigaciones sobre historia de la filosofía. Este tipo de trabajo se ha enfocado sobre todo en análisis de obras de la filosofía antigua, aunque también hay algunas, en menor medida, referidas a autores y corrientes de la modernidad y la época contemporánea. 
De esta manera, se encuentran trabajos como el de François Gagin (miembro del grupo de investigación en ética y filosofía política de la Universidad del Valle) (2011), quien hace referencia a la preocupación que suscitó en la antigüedad la reflexión sobre la muerte como aquella que marca el punto de partida desde el cual es posible descifrar, retrospectivamente, el entramado filosófico de una vida estética y éticamente filosófica. Esto es, cómo mediante la meditación sobre la muerte es posible comprender la vida como el arte de vivir, cómo disponerse para una vida feliz. Todo esto a la luz de la figura de Sócrates como ejemplo claro de una existencia auténticamente filosófica.

Ahora bien, el carácter terapéutico de la filosofía antigua es analizado por Carolina Montoya (2008), quien interpreta la filosofía como terapia o como medicina que cura el alma. Examinando las figuras de Sócrates y Séneca como referentes de la concepción de filosofía como forma de vida hadotiana, Montoya muestra cómo en ambos casos se hace referencia a la filosofía que no admite un saber sin más, sino a la labor filosófica que se completa en la práctica de unos ejercicios particulares que comprometen y transforman la vida completamente como, por ejemplo, la meditación sobre la muerte.

Sandra Romero (2010) se propone rastrear en La República de Platón aspectos propios de la vida filosófica, partiendo del análisis a las tres formas de vida que muestra Platón en el libro IX del texto mencionado, para posteriormente reflexionar sobre la figura de Sócrates y la manera en la que encarna una vida filosófica; esto a la luz de la Apología, el Fedón y Banquete de Platón. Romero analiza el ethos socrático y la mejor forma de vida señalada en La República, con el fin de comparar y mostrar si se relacionan en algún sentido o de qué manera difieren una de la otra. Por otra parte, Luisa Fernanda Rojas (2014) analiza la ironía socrática a partir de la perspectiva de Kierkegaard en su obra Sobre la ironía. Rojas concluye que, siguiendo la figura socrática, la ironía es el camino para la realización de una vida filosófica.

La propuesta de Hadot admite que, además de Sócrates, se puede reconocer en otras figuras de escuelas helenísticas, como el estoicismo, características particulares que develan una coherencia entre discurso y práctica filosófica. Así, por ejemplo, se intenta analizar la obra y pensamiento de filósofos como Séneca, a la luz de la filosofía como un modo de vida. Por ejemplo, la reseña que nos ofrece la profesora Luz Gloría Cárdenas del libro ¿Una ética en tiempos de crisis? (2004) de François Gagin, muestra cómo el autor realiza un trabajo de investigación netamente histórico en el que da cuenta de los aspectos constitutivos de dos escuelas helenísticas, a saber: el estoicismo 
y el epicureísmo, estableciendo también diferencias entre ambas escuelas y mostrando la influencia que tuvieron en nuestra concepción de la filosofía como forma de vida.

Andrea Lozano (2013) busca comprender cómo en la obra de Séneca se refleja la filosofía en tanto práctica. La autora reconoce la escritura senequiana como una suerte de terapia que cura y a su vez transforma la vida de quien padece una enfermedad del alma. Según ella, la terapia filosófica tiene como fundamento corregir o reemplazar las creencias de quien está atormentado por la enfermedad y que determinan su forma de actuar. Lozano permite comprender en qué sentido la enfermedad es responsabilidad de quien la padece y, de igual manera, la forma en la que esta persona llega a la filosofía y, a partir de esta, reestructura su vida.

Igualmente, Lozano (2013) hace referencia al carácter terapéutico que adquiere la escritura como práctica filosófica en la antigüedad. Hace referencia a la escritura de Platón en sus diálogos, así como de las epístolas escritas por Séneca y Epicuro, analizando las particularidades que caracterizan la escritura en tales pensadores, así como los recursos retóricos usados para generar en el lector un reconocimiento en los temas y situaciones abordados en las cartas, así como cierta familiaridad con la vida de quien escribe, como en el caso de Séneca.

En esta misma línea de estudio, Juan Camilo Betancur (2014) va más allá de una reconstrucción de la obra o pensamiento senequiano desde la propuesta de Hadot y analiza el lugar de los recursos literarios y retóricos usados en la terapia filosófica por la que propende Séneca. De este modo, concede especial relevancia al lenguaje terapéutico usado por el pensador romano para hacer efectivo el proceso terapéutico de aquel que padece una enfermedad del alma. Así, Betancur toma en consideración elementos literarios de los que hace uso Séneca para el tratamiento de una enfermedad o bien leve, o bien crónica, para dar lugar a unos ejercicios espirituales que posibiliten llevar una vida filosófica.

Como ya lo vimos, este carácter terapéutico de la escritura se reconoce en la filosofía antigua en autores como Séneca. Sin embargo, es posible rastrear argumentos de este tipo en autores de otras épocas como bien lo muestra Angélica Hernández (2007), quien realiza una interpretación de los Ensayos de Montaigne para justificar la dimensión práctica de la escritura que posibilita un autoconocimiento, y una comprensión del mundo y de la forma en la que nos relacionamos con él. Esto a partir de un examen 
de la propia experiencia de sí mismo que no concibe una actitud contemplativa, sino que propende por una actitud activa y formativa con respecto a la vida misma.

El artículo de Hernández da cuenta de que la noción de filosofía como forma de vida también se puede evidenciar en la obra de filósofos modernos. Así mismo, Germán Meléndez (2014) propone como trasfondo la pregunta por la constitución de la filosofía como arte de vivir en la antigüedad y como trasformación estética de sí mismo, propuesta por algunos autores modernos. Esta pregunta se contextualiza de acuerdo con el tratamiento que Epícteto le da a la misma en sus Disertaciones, y expone cómo se compara a la filosofía con las otras artes para analizar cómo la misma se constituye como una actividad estilística de impronta individual. Lo anterior lleva a concluir que la propuesta de Epícteto es cercana a la noción de arte individualista de vivir, que Alexander Nehamas atribuye a filósofos como Montaigne, Nietzsche y Foucault.

Queda decir que el reconocimiento de un carácter práctico y terapéutico de la filosofía no escapa de la posición escéptica. David Bernal Vera (2011) relaciona el escepticismo de sexto empírico con una actitud que insiste en la investigación. Tal actitud implica la adopción de determinadas prácticas cotidianas que constituían una forma de vida escéptica. A partir de una lectura de los Esbozos pirrónicos, Bernal Vera reconstruye y caracteriza el escepticismo y expone, entre otras cosas, que está vinculado con una apertura a la investigación con un carácter terapéutico que se consideraba una facultad a desarrollar. Concluye, finalmente, que en el escepticismo la práctica no está supeditada a máximas ya determinadas. En su lugar señala que la forma de vida escéptica se constituiría desde la actitud investigativa que le constituye y no de un discurso que le precede.

\section{La filosofía como forma de vida y la preocupación por la formación en la actualidad}

De la mano de la propuesta hadotiana y con los trabajos enunciados no solo se puede reflexionar sobre la manera en que estudiamos la filosofía, sino también sobre la pertinencia de su rol dentro del ámbito formativo. El punto de atención dentro de todo esto es que se rescata la visión antigua de la filosofía, en donde el filósofo es quien lleva una vida conforme a sus principios. No se rechaza el trabajo exegético y académico, pero se propone que incluso esto debe estar permeado por la invitación de los antiguos a transformarse a sí mismo. La filosofía no debería entenderse como 
una disciplina que pretende constituir un lenguaje especializado para unos pocos. La filosofía, siguiendo la concepción del antiguo mundo helenístico y romano, es un ejercicio, una práctica constante; constituye un arte de vivir, una forma de vida.

En la línea de esta problemática expuesta por Hadot, el profesor Óscar Orlando Espinel Bernal (del grupo de investigación Pensamiento, Filosofía y Sociedad, de la Universidad Minuto de Dios) (2014) retoma al autor para mostrar cómo la filosofía en el mundo antiguo era concebida como una práctica pedagógica que pretende guiar la conducta y formar el espíritu de los individuos, lo que lleva a la elección de una forma de vida. Espinel muestra la cercanía de esta propuesta con las prácticas de sí sobre sí de la estética de la existencia de Michel Foucault. La idea del autor colombiano es proponer una vuelta a esta concepción de la filosofía, en donde la misma tiene un sentido ético-formativo. Esto ante un academicismo y una reducción a la teoría que denuncia como predominante en la enseñanza de la filosofía en los centros de estudio contemporáneos, lo que la hace parecer una disciplina tediosa y un asunto distante de la cotidianidad.

La tendencia academicista de la filosofía es descrita como una transmisión de información que se impone sobre la posibilidad de pensar por sí mismo. Espinel denota cómo el filósofo francés Michel Foucault (1994) en Hermenéutica del sujeto estudia a los antiguos desde esta perspectiva, mostrando que la actividad filosófica tiene el propósito de ocuparse de sí mismo, una producción de sí que el filósofo francés identifica como estética de la existencia. La filosofía abre un espacio para conducir la propia vida, es una actividad constante y permanente una vez se emprende. Con todo esto, a lo que se llega es a una conversión de la persona, de su modo de ver el mundo y de darse a él.

Por otro lado, Víctor Florián (2006), situándose en la perspectiva filosófica de Hadot, localiza algunos elementos que se le contraponen a la misma dentro del ámbito de la enseñanza en la actualidad. Florián muestra que se suele incurrir en la mera transmisión de conceptos y teorías para que el estudiante memorice, y que, por otro lado, se tiene la idea de que la filosofía es inútil para la vida y se le ve como un compendio teórico que no suscita mucho interés. Así también, Florián motiva a cuestionarnos la práctica educativa y enuncia aspectos de los que nos podríamos servir para reflexionar al respecto, a saber: el carácter analítico de la filosofía, el cual incentiva una actitud crítica pertinente para el estudiante de educación media; también afirma que la filosofía no es carente de contenidos, por lo que hay que llevar a los estudiantes a 
enfrentarse directamente con textos filosóficos; además, es adecuado que la reflexión se plasme en una producción escrita acerca de los temas estudiados, puesto que a través de la escritura el estudiante da cuenta de la manera en que se ha apropiado de los términos, los textos y la actividad filosófica.

Florián también dice que hay varios frentes en los que hay que seguir investigando, como la construcción de cursos organizados, la selección de temáticas que se enseñen con apoyo de textos y autores relacionados, la elaboración y presentación de textos que sirvan de apoyo para la enseñanza y que se adecúen al nivel formativo de los estudiantes, y finalmente, la creación talleres y seminarios de actualización en materia pedagógica.

Alfredo Durán Mejía (2007) hace una crítica a la filosofía como un servicio y a las implicaciones que ha tenido la academia en las exigencias del quehacer filosófico contemporáneo. El autor propone llevar la filosofía al aula desde una manera distinta a la que comúnmente se realiza y haciendo comprender a los estudiantes que la filosofía no se halla lejos de su propia experiencia vital. El objetivo no es otro que mostrar que la enseñanza de la filosofía debe ser concebida como una experiencia enriquecedora para los estudiantes, en la que ellos mismos descubran la filosofía.

Finalmente, Andrea Lozano (2012) evidencia la necesidad que tenemos quienes nos dedicamos a la educación filosófica a reflexionar sobre nuestro quehacer en un ámbito más allá del académico y formal, para vincularlo a un contexto real que exige más que la interpretación de un discurso filosófico. Por eso, considera pertinente resignificar la actividad de filósofos y educadores en filosofía. Justamente, cultivarse en la filosofía requiere una actitud que nos trasforma a la vez que transforma a otros, de ahí la pertinencia de la labor filosófica en las aulas.

\section{El surgimiento de la práctica filosófica}

Desde la perspectiva expuesta, un factor importante dentro de la inquietud por el papel de la filosofía en el ámbito educativo es el hecho de que se ha ido dejando de lado el carácter vital del ejercicio filosófico a medida que se ha ido enclaustrando en centros académicos formales. Sin embargo, surge una corriente filosófica denominada práctica filosófica o praxis filosófica, la cual busca volver sobre la concepción antigua del ejercicio filosófico. No se niega la influencia de otros autores para esta 
propuesta, pero bien, el análisis hadotiano de los antiguos ha sido uno de los más relevantes. Cabe mencionar algunos trabajos que también han sido importantes en el ámbito de la práctica filosófica. Gabriel Arnaiz (2007), por ejemplo, resalta la labor de Michel Onfray en textos como Las sabidurías de la antigüedad. Contra-historia de la filosofía I (2007). También encontramos a Michel Foucault en El uso de los placeres (2003) y La inquietud de sí (2003), Hermenéutica del sujeto (1994), El gobierno de sí $y$ de los otros (2009) y El coraje de la verdad: el gobierno de sí y de los otros II (2010); Alexander Nehamas con su obra El arte de vivir. Reflexiones socráticas de Platón a Foucault (2005); y Martha Nussbaum con su obra La terapia del deseo. Teoría y práctica de la ética helenística (2003).

Teresa Gaztelu (2014, p. 5) y Mónica Cavallé (2009, pp. 21-22) ubican el surgimiento de la práctica filosófica en 1981 con el filósofo alemán Gerd Achembach, quien introduce la noción de philosophische praxis und beratung (práctica filosófica, asesoramiento filosófico, consulta u orientación filosófica). Noción con la que denominó la actividad que emprendió en un espacio en donde ofrecía un servicio de consulta privada en el que ayudaba a otros en la reflexión sobre su propia vida. Lo que se buscaba orientarse hacia el ejercicio de filosofar, más que al estudio teórico. Esta práctica se fue extendiendo por todo el mundo y, de esta manera, ha ido ampliando y diversificando las formas en que se lleva a cabo.

En esta actividad, según Brenifier (2007), por medio del diálogo se orienta a un consultante a hacerse consciente de los presupuestos de su pensamiento para luego proceder a hacer un análisis crítico de ellos y proponer conceptos que logren expresar el resultado de la reflexión (p. 219). La guía del filósofo asesor no debe caer en la transmisión de información sobre teorías filosóficas, sino que debe acompañar a quien lo consulta en un proceso de indagación filosófica, siguiendo la mayéutica socrática.

Achembach (s.f.), insiste en que al consultante se le debe tomar como un ser único, por lo que se le debe orientar por su propio camino. La formación en filosofía del asesor solamente implica el desarrollo de unas habilidades que le permiten orientar al otro, a saber, de análisis e interpretación, y también el desarrollo de una sensibilidad mayor ante hechos y juicios que pueden pasar desapercibidos. Los espacios de la práctica filosófica buscan ir más allá del ámbito académico formal y generar espacios formativos no oficiales en los que sea posible encontrarse con un público menos limitado. Con este tipo de ejercicios se espera romper con la dicotomía entre 
teoría y praxis esperando que la misma actividad de reflexión de la filosofía involucre el carácter transformador de la misma.

Es menester tener en cuenta que la práctica filosófica no solo se limita a la consulta, han emergido diversos espacios en los que se crean ejercicios con propósitos base de esta corriente. Tanto Gabriel Arnaiz (2007, p. 3) como Juan Domingo Machado y Mónica Cavallé (2009, pp. 14-15) delimitan otros ámbitos en los cuales es posible concebir la orientación filosófica. Hay un ámbito lúdico que se destaca por actividades como cafés filosóficos -tertulias orientadas por un filósofo acerca de un tema que interpele a los participantes- y talleres filosóficos -encuentros de diálogo filosófico con menos asistentes y mayor intervención del orientador-. También está el ámbito que Arnaiz denomina formativo, el cual se desarrolla en organizaciones y entidades de carácter público o privado para clarificar los fines y medios de las mismas. Y finalmente, enuncian el ámbito mediático que se sirve de la difusión en los medios de comunicación.

\section{Estudios y aplicaciones de la práctica filosófica en Colombia}

Las actividades y estudios en torno a la práctica filosófica no son ajenas a nuestro país, aunque no son difundidas con mucha fuerza. Existen trabajos y propuestas de personas directamente vinculadas a la corriente inaugurada por Achembach, $y$ también algunas con bases similares que han emprendido ejercicios que pretenden rescatar el carácter activo de la filosofía.

Cabe destacar el trabajo de Víctor Raúl Jaramillo, quien en su libro Filosofía como medicina. Una mirada hermenéutica (2005), recoge varios escritos en los que se evidencia gran parte de su trabajo y sus aportes para la práctica filosófica en el país. Esta obra no tiene una estructura sistemática y lineal, incluso, contiene textos poéticos. Jaramillo propone a la hermenéutica como guía de investigación y estructuración de los textos que allí expone. Las cuestiones por el habla, el lenguaje y el diálogo son transversales a todo el texto de Jaramillo. Algunos apartados están dedicados a plasmar las reflexiones acerca de lo que el autor llama terapia dialógica, la cual aplica desde la consultoría filosófica. Así mismo, se proponen brevemente lineamientos que aconseja para el quehacer del terapeuta. Se argumenta que el diálogo que se establece en la terapia, en una búsqueda de conocimiento que se emprende, ayuda a 
comprender lo que ocurre y propicia una actividad creadora en donde el consultante se hace partícipe de ello.

Entre tanto, la Escuela de Formación Avanzada y el Círculo de Estudio sobre Filosofía como Medicina del Grupo de Dolor y Cuidado Paliativo de la Universidad Pontificia Bolivariana, edita el número 14 de la revista Cuadernos de formación avanzada. Esta edición de la revista gira en torno a la defensa de la tesis doctoral de Víctor Raúl Jaramillo titulada Terapia dialógica: introducción al consultorio filosófico, la cual cuenta con textos de varios autores.

En el primer escrito de la revista enunciada, Jaramillo (2002) parte de la relación de la palabra con el ser humano, el cual es un ser de acción verbal y de silencio. Jaramillo defiende la existencia de una dimensión terapéutica de la filosofía a través del uso de la palabra, punto del que se sirve para proponer su idea de la terapia dialógica aplicada en su Consultorio Filosófico. El autor propone concebir al filósofo como un puente que permite una apertura de horizontes de quien acude a él. También define a la filosofía como el reconocimiento que el ser humano hace de sí mismo, por lo que la filosofía constituye una actividad vivificadora y liberadora del pensamiento encaminada a la transformación y a la creación.

A partir de la defensa de la tesis doctoral de Jaramillo diversos autores realizan algunos escritos en donde reflexionan sobre determinados aspectos al respecto. Hugo Urrego (2002) centra su atención en la función del diálogo como posibilidad de relación del ser humano con el mundo y con sí mismo. Armando Velásquez (2002) destaca que la discusión en la terapia filosófica pone en tensión diversas formas de ver el mundo propiciando una reflexión acerca de las mismas en un choque de horizontes que genera nuevas posibilidades de ser.

Por otro lado, Alexis Vélez Rodríguez (2002) retoma las nociones principales de la terapia dialógica centrándose en los cambios que esta conlleva en la acción y reflexión acerca de uno mismo y el mundo. Finalmente, Óscar Fernando Acevedo (2002) retoma de Jaramillo el valor de la filosofía como una actividad curadora, y expone algunas características constitutivas de la consultoría filosófica. Este último autor habla del desarrollo de una ética del amor por parte del filósofo frente a su consultante al tomarlo como un sujeto vivo y no como enfermo. 
La labor de la práctica filosófica en el país no se detiene aquí. Hernán Bueno Castañeda (fundador de la Fundación Filosofarte dedicada a la práctica y el asesoramiento filosófico) (2008) hace un acercamiento a la asesoría filosófica desde el conflicto social del país. En el texto se argumenta que quienes se encuentran dentro de sociedades en condiciones de vulnerabilidad tienen una experiencia de alienación y de indefensión. En este punto, expone el autor, es donde el ejercicio de la asesoría filosófica es pertinente, pues, aunque se actúe sobre un tejido histórico que en alguna medida nos condiciona, la reflexión sobre nuestra realidad en el presente da apertura a nuevos rumbos de acción posibles.

Asimismo, Bueno Castañeda (2011), siguiendo a Michel Onfray, hace mención de algunas escuelas de la filosofía antigua de las que rescata el carácter terapéutico de la filosofía, que se da mediante una apertura al diálogo y con una preocupación por el cuidado y el encuentro con el otro. El autor también expone la necesidad que tiene la filosofía de romper barreras epistémicas con el propósito de dialogar con otros saberes.

La puesta en diálogo de la práctica filosófica con diversas corrientes filosóficas también se hace presente en otras investigaciones. Martha Patiño (vinculada a la Asociación Americana de Practicantes Filósofos y parte del grupo de investigación Fray Saturnino Gutiérrez, O. P. Filosofía de la educación en Colombia y América Latina de la Universidad Santo Tomás) (2014) plantea que el sufrimiento es un activador de la creación, poniendo en comunión el concepto de creatividad de John Dewey. Tal concepto se relaciona con una noción de ser humano como creador artesano de sí mismo , esto es, la idea de creación de la propia existencia y transformación de sí mismo a la que conlleva el ejercicio interpretativo de la práctica filosófica.

Sumado a lo anterior, en octubre de 2015 en la Universidad Pontificia Bolivariana se llevó a cabo el VIII Seminario Nacional de Familia. El ser del terapeuta: dimensiones ética, estética, política y profesional, organizado por el Nodo Antioquia de la Red de Programas Universitarios en Familia, en donde se expusieron presentaciones acerca del ejercicio clínico y la labor del terapeuta. Aunque, en su mayoría, las presentaciones en el evento fueron más cercanas a la psicoterapia, la pregunta por el cuidado de sí fue tema constante y, de la misma manera, hubo acercamientos a la reflexión filosófica desde el ejercicio médico.

En el evento enunciado, Iván Darío Carmona Aranzazu (miembro del grupo de investigación Epimeleia de la Universidad Pontificia Bolivariana de Medellín) (2015), 
retomando la perspectiva hadotiana, vuelve sobre nociones del estoicismo y del epicureísmo y pone en relación la práctica médica con el cuidado de sí característico de la filosofía. Se argumenta que el filósofo es un terapeuta que somete a examen a la palabra, discursos y actos morales para hacer más comprensibles las tensiones de la existencia. En el marco del mismo evento, María Victoria Builes Correa (2015), médica y terapeuta de pareja y familia, retoma a Michel Foucault y propone tratar el problema médico del agotamiento -caracterizado por un proceso de vacío físico y emocional derivado de diversas situaciones personales, laborales y sociales- a partir de la noción de cuidado de sí, pues le pondría límites y lo podría direccionar de manera que haya una posibilidad de hacer una mejor existencia.

Ahora bien, estos desarrollos se han dado también en el ámbito de la educación. Miguel Ángel Gómez Mendoza (pertenece al grupo de investigación en Educación y Pedagogía de la Universidad Pedagógica y Tecnológica de Colombia) (2008) hace un rastreo de diversas prácticas y paradigmas dominantes en el campo de la enseñanza de la filosofía para mostrar que se vive una crisis fundamentada en una ausencia de reflexión en cuanto a la didáctica y la falta de nuevas propuestas de enseñanza. Tras hacer una breve mención al movimiento práctica filosófica, siguiendo a Michel Tozzi, se propone la discusión de tipo filosófico como práctica alternativa, delimitando sus características, su papel en la enseñanza de la filosofía, sus condiciones de posibilidad y sus desafíos en su inmersión en el campo educativo.

En este mismo contexto encontramos los clubes de lectura Leer los filósofos llevados a cabo en la ciudad de Medellín, liderados por la profesora Luz Gloria Cárdenas Mejía (pertenece al grupo de investigación Filosofía y Enseñanza de la Filosofía). Como resultado de esta experiencia se publicó el texto Nomadizar la filosofía. Propuesta para el fomento de la lectura de la filosofía (2014) de Carolina Villada Castro, y también Leer con otros. Nomadizar la filosofía, un paraje de (2014) con la autoría de Carolina Villada Castro, Hernán Alonso Jaramillo, Luisa Fernanda Castaño, Luz Gloria Cárdenas Mejía y Yon Leider Restrepo Monsalve.

Estos textos cuentan cómo se construyó la propuesta, la forma en que se realizó, se relatan experiencias de quienes hicieron parte de ello y, a su vez, proponen una guía para la realización de clubes de este tipo. De esta manera, se dan recomendaciones acerca de la selección de temas y autores, la divulgación y los lugares de encuentro. Los escritos también muestran que el espacio se crea con la idea de nomadizar la filosofía, llevándola fuera del ámbito académico propiciando el enriquecimiento de su estudio 
desde las inquietudes de las personas acerca de su propia vida y su cotidianidad. Lo anterior con la idea de que en la filosofía se puede ser un espacio de encuentro con otros. Los clubes de lectura se realizaron en bibliotecas públicas y fueron abiertos al público general, guiados por un promotor que diseñaba y guiaba las actividades de lectura de acuerdo con el contexto vital del club que estaba a su cargo.

También se han desarrollado trabajos similares en la ciudad de Bogotá. Víctor Andrés Rojas (2009) da cuenta de la labor pedagógica desarrollada durante el 2006 en el Centro Educativo Amigoniano. Escuela de Trabajo El Redentor. Rojas cuenta cómo se realizó una labor pedagógica sustentada en la filosofía para niños ( $\mathrm{FpN})$, propuesta retomada del filósofo estadounidense Matthew Lipman. La FpN es tomada como un recurso didáctico para acercar a los jóvenes a la reflexión y el diálogo filosófico, a partir de sus experiencias de vida particulares en la comunidad de indagación. El trabajo pedagógico ejecutado en dicho lugar posibilitó a quienes lo llevaron a cabo comprender que la propuesta de Lipman va más allá de la forma habitual de abordar la filosofía en contextos educativos no formales, promoviendo el pensamiento autónomo, la reflexión y el diálogo entre jóvenes, así mismo, posibilita que ellos mismos descubran y comprendan el mundo y su labor en él desde una perspectiva completamente renovada.

Finalmente, en abril de 2008 se llevó a cabo en la Fundación Universitaria Luis Amigó el I Congreso Internacional de Filosofía: filosofía como medicina, coordinado por el profesor Víctor Raúl Jaramillo. En este evento se trataron temas acerca de la asesoría filosófica, la medicina y la palabra, la práctica filosófica, entre otros; y contó con la participación de exponentes internacionales cuyos trabajos se han destacado en el tema en cuestión, como Mónica Cavallé, Óscar Brenifier, Ran Lahav, entre otros (Ministerio de Educación Nacional, 2008). También es pertinente enunciar que la American Philosophical Practitioners Assosiaton, corporación educativa que promueve la práctica de la asesoría filosófica, reconoce a tres colombianos como miembros ${ }^{1}$, a saber, Martha Patiño, Alejandra Fierro y Camilo Martínez Orozco.

Finalmente, cabe mencionar el ciclo de conferencias y talleres Práctica filosófica: nuevos enfoques, realizado por la Universidad Pedagógica Nacional, la Universidad Santo Tomás, el grupo de investigación Filosofía y Enseñanza de la Filosofía y el ICBF, durante el segundo semestre de 2015. Este evento tuvo como propósito ofrecer una

1 Información tomada de https://www.appa.edu/facilitators.htm 
introducción a la práctica filosófica desde diferentes enfoques y propuestas que han surgido recientemente.

Durante este evento se llevaron a cabo conversatorios, conferencias, talleres, espacios de terapia filosófica, entre otras actividades, las cuales se realizaron con los siguientes invitados: Maximiliano Prada (U. Pedagógica), Diana Acevedo (U. Pedagógica), Ángela Valderrama (U. Pedagógica), Ángela Uribe (UNAL), María Lucía Rivera (U. del Bosque), Luz Gloria Cárdenas (U. de Antioquia), Adriana Urrea (PUJ), Germán Meléndez (UNAL), Andrea Lozano (U. de los Andes), Francisco Rodríguez (U. del Rosario), Natalia Orozco (PUJ), Teresa Gaztelu (Escuela de Práctica Filosófica de Madrid, España), Mercedes García Márquez (Escuela de Práctica Filosófica de Madrid, España) y Óscar Brenifier (Institut de Pratiques Philosophiques, París, Francia).

\section{Conclusiones}

En este artículo hemos intentado exponer los principales planteamientos acerca de la noción de filosofía como forma de vida desde la perspectiva de Pierre Hadot, y acerca del movimiento práctica filosófica para examinar el trabajo realizado al respecto en Colombia. Se descubrió que este campo ha sido poco explorado en el país, pues son pocas las publicaciones que tratan estos temas de manera explícita y la mayoría de investigaciones encontradas son recientes y aún están en desarrollo. De todos modos, se encontró que, dentro de las publicaciones encontradas, las temáticas en cuestión se han tratado de cuatro maneras: 1) con el análisis y problematización teórica de la propuesta de Hadot y de la práctica filosófica; 2) a través del estudio de la historia de la filosofía con un principal enfoque en la filosofía griega y helenista a la luz de la filosofía como forma de vida; 3 ) en el análisis de cuestiones y problemas educativos desde la perspectiva de la filosofía como forma de vida; 4) en la puesta en práctica de ejercicios concretos que se proponen aplicar la teoría acerca de la filosofía como forma de vida y de la práctica filosófica.

Pudimos evidenciar que hay un mayor número de publicaciones sobre los estudios teóricos que se han realizado, y aunque existen propuestas de carácter práctico, son pocas las publicaciones al respecto. Por lo anterior, es necesario ampliar las vías de comunicación y de divulgación de estas nuevas propuestas, así como entablar diálogos y promover proyectos entre quienes han investigado sobre estos temas. 
La mayoría de prácticas filosóficas rastreadas se dan en espacios educativos no formales y se han dado en relación con la terapia vinculada al espacio psiquiátrico y médico. Aun cuando ha habido aproximaciones por acercar la práctica filosófica al campo de la enseñanza de la filosofía, se puede constatar que aún queda abierta la pregunta por el modo en que dichas prácticas puedan servir a la enseñanza de la filosofía en espacios de educación formal, más allá, por supuesto, de las propuestas de FpN. También es pertinente emprender investigaciones que logren articular el estudio teórico de la propuesta con su aplicación práctica y, especialmente, que puedan ser desarrolladas dentro del ámbito educativo. Esto último sería oportuno en la medida en que se intentaría borrar la brecha entre lo teórico y lo práctico desde la misma investigación filosófica y pedagógica.

\section{Referencias}

Acevedo, o. "El lugar del amor, de los principios y de la palabra en la Consultoría Filosófica”. En: Colombia Cuadernos De Formación Avanzada. Ed: 14 p.44 - 49.

Achembach, G. (s.f.). Breve respuesta a la pregunta: ¿Qué es la orientación filosófica? En A. Gabriel (Trad.). El Búho, Revista Electrónica de la Sociedad Andaluza de filosofía, (9).

Aranzazu, C. (2015). Cuidado de sí y terapia: un guiño filosófico. Memorias del VIII Seminario Nacional de la Familia. Medellín: Universidad Pontificia Bolivariana. Recuperado de http://www.upb.edu.co/pls/portal/docs/PAGE/ GPV2_UPB_MEDELLIN/PGV2_M030_PREGRADOS/PGV2_M030040040_ TRABAJOSOCIAL/MEMORIAS\%20EVENTO\%20RED\%20(RESUMENES).PDF

Arnaíz, G. (2007a). ¿Qué es la filosofía práctica? A Parte Rei. Revista de filosofía, 53.

Arnaíz, G. (2007b). Relevancia de las aportaciones de Pierre Hadot y Michel Onfray para la filosofía práctica. A Parte Rei. Revista de filosofía, 52.

Bernal, D. (2011). El escepticismo pirrónico: la investigación como forma de vida (trabajo de grado). Bogotá: Pontificia Universidad Javeriana. Recuperado de http:// repository.javeriana.edu.co/bitstream/10554/6805/1/tesis145.pdf 
Betancur, J. C. (2014). La terapia de la palabra. Una lectura de las epístolas morales a Lucilo de Séneca. Memorias del Congreso Colombiano de Filosofía. V Congreso (Medellín, 28 de julio - 1 de agosto de 2014). Sociedad Colombiana de Filosofía.

Brenifier, O. (2007). ¿Puede la filosofía convertirse en una práctica? Diálogo filosófico, 68, (pp. 217-228).

Bueno, H. (2008). Filosofía aplicada en Colombia: consultoría filosófica y realidad nacional. En J. Barrientos Rastrojo (Ed.). Filosofía aplicada y universidad, (pp. 71-81). Madrid: Visión Libros Gestor Editorial.

Bueno, H. (2011). Filosofía y unidad. Una reflexión sobre la asesoría filosófica como quehacer sistémico. Apuntes filosóficos, 20(39), 67-79.

Builes, M. V. (2015). Cuidado y agotamiento del terapeuta. Memorias del VIII Seminario Nacional de la Familia. Medellín: Universidad Pontificia Bolivariana. Recuperado de http://www.upb.edu.co/pls/portal/docs/PAGE/GPV2_UPB_MEDELLIN/ PGV2_M030_PREGRADOS/PGV2_M030040040_TRABAJOSOCIAL/ MEMORIAS\%20EVENTO\%20RED\%20(RESUMENES).PDF

Cárdenas, L. G. (2004). Reseña: Francois Gagin: ¿Una ética en tiempos de crisis? Ensayos sobre estoicismo. Estudios de Filosofía, (30), 153-155.

Cavallé, M. (2009). Diálogos para una vida filosófica. En M. Cavallé y J. D. Machado. Arte de vivir, arte de pensar. Iniciación al asesoramiento filosófico, (pp. 21-82). Bilbao: Editorial Desclée de Brouwer, S. A.

Cavallé, M., y Machado, J. (2009). Prólogo: ¿Qué es la práctica filosófica? En M. Cavallé y J. Machado. Arte de vivir, arte de pensar. Iniciación al asesoramiento filosófico, (pp. 11-19). España: Editorial Desclée de Brouwer, S. A.

Chase, M. (2012). Recordando a Pierre Hadot. En A. Fernando (Trad.). Revista Colombiana de Filosofía, (16), 11-21.

Díaz, J. A. (2001). ¿Filosofía para quién? Ideas y Valores, (117), 79-96.

Durán, A. (2007). Filosofía para no filósofos. Universitas Philosophica, 24(48), 149-164.

Espinel, O. O. (2014). Filosofía, prácticas de sí y arte de vivir. Fermentario, 8(2).

Florián, V. (2006). La posibilidad de la filosofía. Cuestiones de filosofía, (pp. 11-121). 
Foucault, M. (2003). Historia de la sexualidad 3. La inquietud de sí. Buenos Aires: Siglo XXI Editores Argentina, S. A.

Foucault, M. (1994). Hermenéutica del sujeto. Madrid: Ediciones de la Piqueta.

Foucault, M. (2003). Historia de la sexualidad 2. El uso de los placeres. Buenos Aires: Siglo XXI Editores Argentina, S. A.

Foucault, M. (2006). Seguridad, territorio y población. Curso en el Collége de France (1977-1978). Argentina: F.C.E.

Foucault, M. (2009). El gobierno de sí y de los otros. Buenos Aires: Fondo de Cultura Económica de Argentina, S. A.

Foucault, M. (2010). El coraje de la verdad: el gobierno de sí y de los otros II. Buenos Aires: Fondo de Cultura Económica de Argentina, S. A.

Gagin, F. (2011). El horizonte filosófico de la muerte desde la antigüedad. El punto de inflexión para el desciframiento del entramado de una vida filosófica. Praxis Filosófica, (33), 135-152.

Gaztelu, T. (2014). Una lectura del theravāda desde la philosophische lebensberatung. Universidad Complutense de Madrid.

Gómez, M. Á. (2008). La discusión en el campo de la educación y la enseñanza de la filosofía. Revista Colombiana de Educación, (55), 167-187.

Hadot, P. (1998). ¿Qué es la filosofía antigua? México: Fondo de Cultura Económica. Hadot, P. (2006). Ejercicios espirituales y filosofía antigua. España: Ediciones Siruela. Hernández, A. (2007). Mongtaine: la escritura como práctica vital. Cuadrante Phi, (14). Jaramillo, V. R. (2002). Palabra y padecimiento. Defensa de una tesis doctoral. Cuadernos de formación avanzada, (14), 7-24.

Jaramillo, V. R. (2005). Filosofía como medicina: una mirada hermenéutica. Medellín: Editorial Universidad Pontificia Bolivariana.

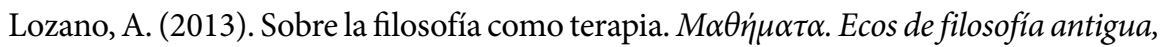
(pp. 477-488). Lima: Fondo Editorial de la Pontificia Universidad Católica del Perú. 
Lozano, A. (2013). Cartas filosóficas: desde la amistad y con sencillez. Estudios Filosóficos, (47), 121-133.

Meléndez, G. (2014). Sobre el arte de vivir en Epícteto. Eidos: Revista de Filosofía de la Universidad del Norte, (20), 271-310.

Meléndez, G. (2015). Filosofía como forma de vida. En L. E. Hoyos y A. Mejía (Eds.). Motivos filosóficos: una introducción temática a la filosofía, (pp. 41-57). Bogotá: Universidad Nacional de Colombia - Universidad Externado de Colombia.

Ministerio de Educación de Colombia (2008). FUNLAM Y Fusa realizaron I Congreso Internaciona de filosofía. Recuperado de: http://www.mineducacion.gov.co/ cvn/1665/w3-article-158194.html

Montoya, C. (2008). "Lectio inauguralis: La Columna rota". En: Colombia Universitas Philosophica ISSN: 0120-5323 ed: Editorial Pontificia Universidad Javeriana v.50 fasc.N/A p. $257-277$.

Nehamas, A. (2005). El arte de vivir. Reflexiones socráticas de Platón a Foucault. Valencia: Pre-Textos.

Nussbaum, M. (2003). La terapia del deseo. Teoría y práctica de la ética helenística. Barcelona: Ediciones Paidós Ibérica, S. A.

Pachón, D. (2014). Pierre Hadot: la filosofía como forma de vida. Le Monde Diplomatique, 11(131), 36-37.

Patiño, M. (2014). Una aproximación pragmatista al problema del sufrimiento desde la práctica filosófica. En L. F. Cardona Suárez. Filosofía y dolor. Hacia la autocomprensión de lo humano, (pp. 597-616). Bogotá: Editorial Pontificia Universidad Javeriana.

Rojas, V. A. (2009). La práctica filosófica como una actividad socialmente comprometida: diálogo filosófico y desarrollo de la autonomía en un contexto de reeducación de menores infractores. Cuestiones de Filosofía, (pp. 69-85).

Rojas, L. F. (2014). La ironía socrática como forma de vida. (Tesis para optar al título de magíster en Filosofía). Bogotá: Universidad Nacional de Colombia. Recuperado de http://www.bdigital.unal.edu.co/46314/1/4438390.2014.pdf 
Romero, S. (2010). La filosofía como forma de vida. Bogotá: Pontificia Universidad Javeriana. Recuperado de http://repository.javeriana.edu.co/bitstream/10554/ 6765/1/tesis81.pdf

Urrego, H. (2002). Conciencia y revelación. Reflexión sobre la naturaleza del diálogo, a partir de la defensa de la tesis doctoral de Víctor Raúl Jaramillo. Cuadernos de formación avanzada, (14), 25-29.

Velásquez, A. (2002). Reconfiguración del yo en la terapia dialógica. A partir de la defensa doctoral de Víctor Raúl Jaramillo. Cuadernos de formación avanzada, (14), 30-31.

Vélez, A. (2002). Comentario a la defensa de la tesis doctoral sobre terapia dialógica o la filosofía como medicina de Víctor Raúl Jaramillo. Fundamentación de un consultorio filosófico por medio del lenguaje. Cuadernos de formación avanzada, (14), 38-43.

Villada, C. (2014). Nomadizar la filosofía. Propuesta para el fomento de la lectura de la filosofía. Medellín.

Villada, C., Jaramilloleta, H. A., Castaño, L. F., Cárdenas, L. G., y Restrepo, Y. L. (2014). Leer con otros. Nomadizar la filosofía, un paraje. Una guía para la realización de clubes de lectura en filosofía. Medellín: Universidad de Antioquia.

Zabaleta, S. (1977). La filosofía política de la conquista. México: F.C.E. 